\title{
Mitochondrial noncoding RNA transport
}

\author{
Kyoung Mi Kim, Ji Heon Noh ${ }^{*}$, Kotb Abdelmohsen \& Myriam Gorospe \\ Laboratory of Genetics and Genomics, National Institute on Aging Intramural Research Program, National Institutes of Health, Baltimore, \\ Maryland 21224, USA
}

\begin{abstract}
Mitochondria are cytosolic organelles essential for generating energy and maintaining cell homeostasis. Despite their critical function, the handful of proteins expressed by the mitochondrial genome is insufficient to maintain mitochondrial structure or activity. Accordingly, mitochondrial metabolism is fully dependent on factors encoded by the nuclear DNA, including many proteins synthesized in the cytosol and imported into mitochondria via established mechanisms. However, there is growing evidence that mammalian mitochondria can also import cytosolic noncoding RNA via poorly understood processes. Here, we summarize our knowledge of mitochondrial RNA, discuss recent progress in understanding the molecular mechanisms and functional impact of RNA import into mitochondria, and identify rising challenges and opportunities in this rapidly evolving field. [BMB Reports 2017; 50(4): 164-174]
\end{abstract}

\section{INTRODUCTION}

As a primary site for energy generation, calcium signaling, and apoptotic factors, mitochondria are essential multifunctional organelles in eukaryotic cells. Derived from endosymbiotic bacterial ancestors, eukaryotic mitochondria have their own genome and are equipped with fully functional gene expression machineries $(1,2)$. To maintain their biogenesis and function, however, mitochondria require a large number of proteins that are encoded by the nuclear genome, translated in the cytosol, and subsequently imported into mitochondria. Accordingly, there has been a strong effort to elucidate the mechanisms of protein import into mitochondria (3-5).

Since the first discovery of cytosolic transfer RNAs (tRNAs) in mitochondria 50 years ago (6), evidence has accumulated supporting the notion that many types of RNA transcribed from nuclear DNA are actively delivered to mitochondria.

*Corresponding author. Tel: +410-558-8698; Fax: +410-558-8331; E-mail: jiheon.noh@nih.gov

https://doi.org/10.5483/BMBRep.2017.50.4.013

Received 19 January 2017

Keywords: Cellular transport, Mitochondria, Mitochondrial function, Noncoding RNA
Imported RNAs include different types of noncoding (nc)RNAs, such as tRNAs, $5 S$ rRNA, MRP RNA (RMRP), and RNase P RNA (RPPH1) (7-13), as well as microRNAs (mitomiRs) (14, 15). Their mobilization into mitochondria requires a range of protein factors (16-19), although the mechanisms that select and import RNAs into mitochondria, as well as the impact of imported RNAs on mitochondrial gene expression programs are largely unknown.

\section{MITOCHONDRIAL TRANSCRIPTS AND THEIR FUNCTIONS}

The mammalian mitochondrial genome $(\sim 12,500 \mathrm{bp})$ encodes 13 proteins, 22 tRNAs, and two ribosomal RNAs, 125 rRNA and $16 S$ rRNA, which assemble the small (28S) and large (39S) subunits of the $55 \mathrm{~S}$ mitochondrial ribosome (20). The mammalian mitochondrial DNA is transcribed as polycistronic precursor RNAs synthesized from both strands, the heavy $(\mathrm{H})$ and the light (L) strands. The individual mitochondrial (mt-) rRNA and mt-mRNA sequences are regularly interspersed with mt-tRNA genes (21). The 13 proteins encoded by the mitochondrial genome function in the oxidative phosphorylation (OX-PHOS) complex $(21,22)$.

\section{IncND5, IncND6, and IncCyt b}

Recently, two different laboratories reported whole-transcriptome analyses of purified human mitochondria and mitoplasts $(23,24)$. The studies identified numerous small RNAs and long noncoding RNAs (IncRNAs) transcribed from the mitochondrial genome. Among them, the IncRNAs IncND5, IncND6, and IncCyt $b$ were identified as the counterpart antisense transcripts of the mitochondrial ND5, ND6, and CYTB mRNAs, respectively (23). IncND5 and IncCyt $b$ were transcribed from the $\mathrm{L}$ strand, and IncND6 from the $\mathrm{H}$ strand of the mitochondrial DNA. Intriguingly, one of the known mitochondrial RNA processing proteins, the mitochondrial RNase $\mathrm{P}$ protein 1 (MRPP1) (25), was proposed to have a unique RNA processing function, influencing the maturation and abundance of IncRNA transcripts (24) (Table 1).

\section{SncmtRNA and ASncmtRNAs}

Villegas et al. reported a human mitochondrial chimeric transcript called sense noncoding mitochondria ribosomal RNA (SncmtRNA) (26), structurally similar to a mouse 
mitochondrial RNA, containing a loop structure and a long inverted repeat (IR) linked to the $5^{\prime}$ end of the sense mitochondrial 165 rRNA (27). Interestingly, SncmtRNA was only detected in proliferating cells but not in resting cells, suggesting that it might be a marker of cell proliferation (26). Two other ncRNAs that formed a similar chimeric structure with SncmtRNA but containing the antisense fragment of $16 \mathrm{~S}$ rRNA transcribed from the L-strand were later identified (ASncmtRNA1 and ASncmtRNA2) (28).

Unlike the SncmtRNA, which is abundant in normal and cancerous proliferating cells, ASncmtRNA1 and ASncmtRNA2 were found expressed only in normal cells and were much less abundant in tumor cells $(26,28)$. A striking subsequent report found that both SncmtRNA and ASncmtRNAs were found in the nucleus associated with heterochromatin, suggesting the possibility that these mitochondrial IncRNAs participate in intraorganellar communication via retrograde signaling path- ways (29). Recently, the ASncmtRNA2 was found upregulated in senescent cells, where $A S n c m t R N A 2$ delayed cell cycle progression through the $\mathrm{G} 2 / \mathrm{M}$ cell cycle phases, possibly through the action of two microRNAs (hsa-miR4485 and hsa-miR1973) derived from ASncmtRNA2 (30) (Table 1, Fig. 1).

\section{LIPCAR}

Very recently, a novel mitochondrial DNA-encoded IncRNA, LIPCAR (long intergenic noncoding RNA predicting cardiac remodeling) was identified in plasma of patients with left ventricular (LV) remodeling post-myocardial infarction (MI). LIPCAR levels declined in early stages after myocardial infarction, but increased in late stages, coinciding with LV remodeling. Increased levels of LIPCAR identified patients at high risk of heart failure or death, suggesting that LIPCAR was a possible biomarker for cardiac remodeling in patients who had an episode of acute MI (31) (Table 1, Fig. 1).

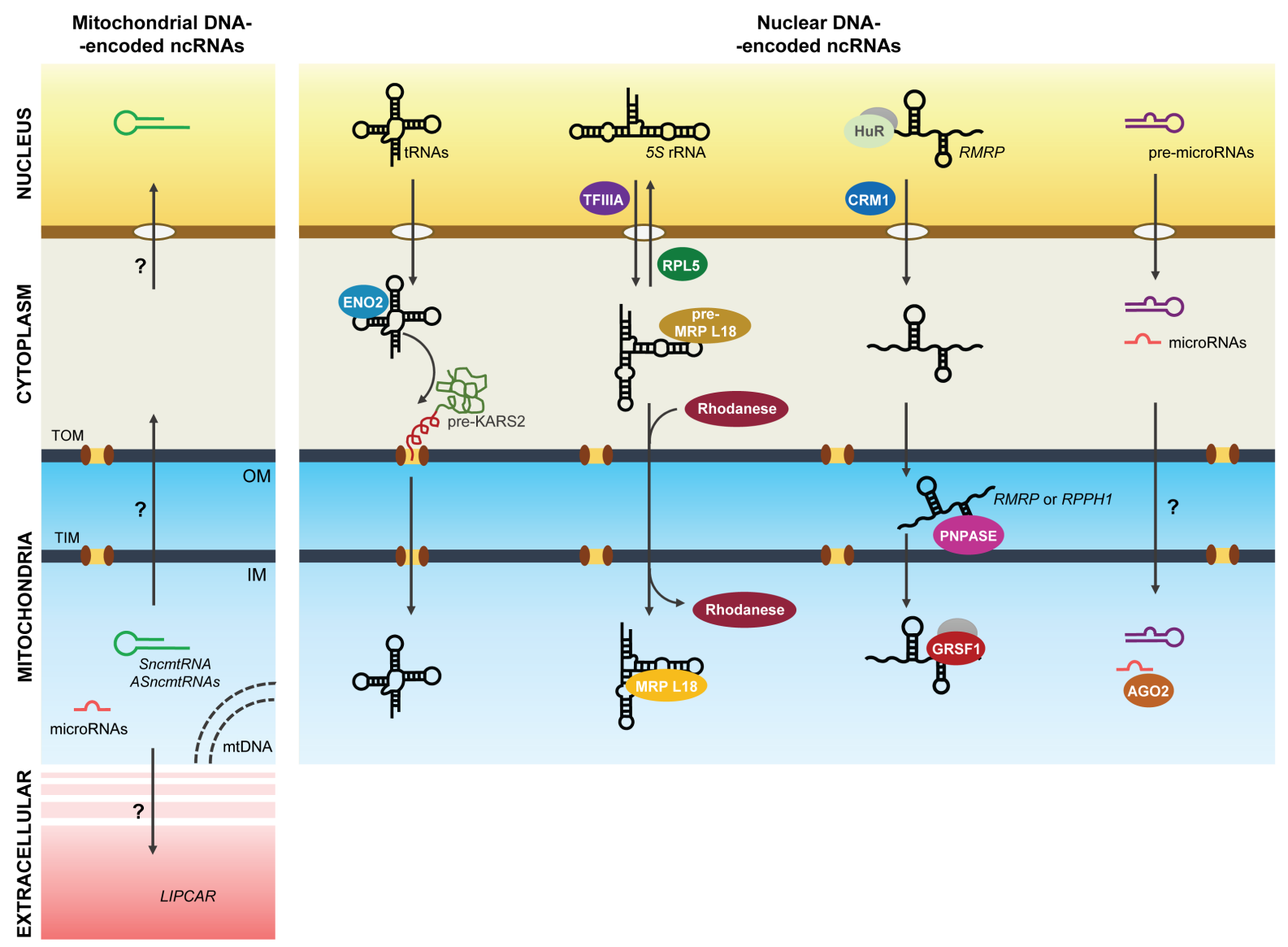

Fig. 1. Schematic of the ncRNAs transported through the mitochondrial outer and inner membranes (OM, IM). Left, ncRNAs synthesized in mitochondria but found elsewhere (nucleus and extracellular space); right, ncRNAs synthesized in the nucleus and found in mitochondria, including tRNAs, $5 S$ rRNA, IncRNAs like $R M R P$, and microRNAs and their precursors. Transport factors identified as mediators of these processes, including many ncRNA-interacting RBPs, are indicated. Yellow, nucleus; gray, cytoplasm; blue, mitochondria; pink, extracellular space. 


\section{LOCALIZATION OF NUCLEAR DNA-ENCODED RNAs INTO MITOCHONDRIA}

Although mitochondria synthesize dozens of RNAs from their own mitochondrial DNA, some nuclear DNA-encoded RNAs can be mobilized into mitochondria. As mentioned above, the import mechanism of cytoplasmic proteins into mitochondria has been studied in detail, but the mechanisms that import nuclear DNA-encoded RNAs into mitochondria are far less clear (32). Only a few noncoding transcripts are selectively transported into the mitochondrial matrix $(17,24)$. In this section, we discuss the current knowledge of the major mitochondria-localized ncRNAs: tRNA, $5 S$ rRNA $(9,11)$, MRP RNA (RMRP) (33), RNase P RNA (RPPH1) (12), and microRNAs (mitomiRs) $(14,15)$.

\section{tRNA (tRNA ${ }^{\text {Lys }}$, tRK1)}

The presence of nuclear DNA-encoded tRNAs in mitochondria was first suggested decades ago from a survey of the protozoan Tetrahymena (6). A following study in yeast $S$. cerevisiae also found that one of two mitochondrial tRNAs (isoacceptors of tRNA ${ }^{\text {Lys }}$ ) originated from the nuclear DNA (34). Since this time, the traffic of nuclear DNA-encoded tRNAs to mitochondria has been observed in many organisms, including protozoa (35-40), yeast $(41,42)$, plants (43-48), and mammals (49). We now recognize that in most organisms at least a few cytosolic tRNA species are required for maintaining mitochondrial biogenesis $(50,51)$. Moreover, a cytoplasmic tRNA from yeast (tRNA ${ }^{\text {Lys }}$; tRNALys acceptor 1, tRK1) was found to be internalized into human mitochondria $(32,52)$, suggesting that yeast and human mitochondria might share key components of RNA import (53).

Mitochondrial tRNA import occurs in higher organisms including human, even though mitochondrial DNA already encodes a full set of tRNAs required for the mitochondrial translation $(52,54,55)$. The protein factors responsible for targeting the yeast tRNA tRK1 to mitochondria include the glycolytic enzyme enolase (ENO2P), which binds tRK1 to form a complex that is directed to the mitochondrial surface, whereupon the tRNA is handed over to the precursor of the mitochondrial lysyl-tRNA synthetase (preMSK or pre-LysRS) (56-58). The resulting complex tRNA-pre-LysRS is then internalized into the mitochondrial matrix through the protein import pathway, comprising the translocase of the translocase of the outer (TOM) and inner (TIM) mitochondrial membrane (the TOM/TIM complex) $(59,60)$. A recent study investigating the conformational change of the labeled tRNA ${ }^{\text {Lys }}$ (tRK1) suggested that tRNA alters its structure upon binding to each carrier protein (ENO2P and pre-LysRS) of the import pathway (61). Both tRK1 and an artificial RNA containing the structural elements required for tRK1 mitochondrial import were targeted into human mitochondria with assistance by the cytosolic precursor of human mitochondrial lysyl-tRNA synthetase (pre-KARS2) and mammalian ENO2 (62). However, it is still unclear how tRNA and pre-LysRS remain bound during translocation through the the TOM and TIM complexes $(63,64)$, despite indications that the mitochondrial protein import machinery might accommodate proteins conjugated with RNAs (65) (Fig. 1).

\section{S rRNA}

Ancestral mitochondria were believed to have a complete set of rRNAs, but most mitochondrial rRNAs, including $5 S$ rRNA, were lost during evolution (66). Two proteins were found to regulate the intracellular distribution and ribosomal assembly of $5 S$ rRNA: the transcription factor TFIIIA, which binds to $5 S$ rRNA and mediates the nuclear export of $5 S$ rRNA and its ribosomal integration in $X$. laevis oocytes $(67,68)$, and the ribosomal protein L5, which forms the RNP complex $5 S$ rRNA-L5 (69), essential for delivering $5 S$ rRNA to the cytoplasm and assembling it into ribosomes $(70,71)$ (Fig. 1).

Other studies showed that a substantial portion of nuclear DNA-encoded $5 S$ rRNA is directed to mammalian mitochondria $(9,11)$. Entelis et al. proposed that cytosolic 5S rRNA imported into mitochondria might substitute for its missing counterpart and form a functional mitochondrial ribosome (mitoribosome) large subunit (LSU) (32). Several proteins regulating $5 S$ rRNA import into mitochondria, as well as key $5 S$ rRNA motifs (helix I and helix IV-loop D) have been identified in recent years (72). For example, the mitochondrial enzyme Rhodanese bound the helix I sequence of $5 S$ rRNA and enhanced $5 S$ rRNA import, while Rhodanese depletion abolished $5 S$ rRNA import into mitochondria and decreased global mitochondrial translation, suggesting that the Rhodanese-driven localization of $5 S$ rRNA enhances mitochondrial function (73) (Fig. 1). Human MRP (mitochondrial ribosomal protein)-L18 was identified as another $5 S$ rRNA import factor. As observed for Rhodanese, the import sequence of 5S rRNA directly interacted with mature MRP-L18 but also with its precursor, preMRP-L18, which contains a mitochondria-targeting sequence in its $\mathrm{N}$-terminal region. The interaction between preMRP-L18 and 5S rRNA in the cytosol causes a conformational change in $5 S$ rRNA that makes it recognized by Rhodanese and translocated into mitochondria. In the matrix, the innermost space in mitochondria, $5 S$ rRNA was proposed to associate with the mature MRP-L18 and with mitoribosomes, affecting mitochondrial translation efficiency (18) (Fig. 1).

\section{MRP RNA (RMRP)}

The 267-nt long IncRNA RMRP is an RNA component of the mitochondrial RNA-processing endoribonuclease complex (RNase MRP), and its mutation causes the pleiotropic human disease cartilage-hair hypoplasia $(\mathrm{CHH})(74) . R M R P$ is broadly expressed in mouse and human tissues from an intronless nuclear gene (75), and resides in the nucleus and mitochondria. In the nucleus, $R M R P$ is involved in the $5^{\prime}$ end maturation of $5.8 S$ rRNA $(76,77)$ and influences yeast cell division cycle 
Table 1. Mitochondrial noncoding RNAs

\begin{tabular}{|c|c|c|c|c|c|c|}
\hline $\begin{array}{l}\text { Genome } \\
\text { source }\end{array}$ & Noncoding RNA & $\begin{array}{l}\text { Subcellular } \\
\text { localization }\end{array}$ & $\begin{array}{l}\text { Interacting proteins } \\
\text { (RBPs) }\end{array}$ & $\begin{array}{l}\text { Translocation } \\
\text { Mechanism }\end{array}$ & Functions & References \\
\hline \multirow[t]{7}{*}{$\begin{array}{l}\text { Mitochondri } \\
\text { al DNA }\end{array}$} & IncND5 & Mitochondria & MRPP1 & Unknown & $\begin{array}{l}\text { Mitochondrial gene } \\
\text { expression }\end{array}$ & $(23,24)$ \\
\hline & IncND6 & & & & & \\
\hline & IncCyt b & & & & & \\
\hline & SncmtRNA & $\begin{array}{l}\text { Nucleus, } \\
\text { mitochondria }\end{array}$ & Unknown & & Unknown & $(26,29)$ \\
\hline & ASncmtRNA1 & & & & Unknown & $(28,29)$ \\
\hline & ASncmtRNA2 & & & & Cell cycle regulation & $(28-30)$ \\
\hline & LIPCAR & $\begin{array}{l}\text { Extracellular } \\
\text { (plasma) }\end{array}$ & & & Unknown & (31) \\
\hline \multirow[t]{5}{*}{$\begin{array}{l}\text { Nuclear } \\
\text { DNA }\end{array}$} & tRNA & $\begin{array}{l}\text { Cytoplasm, } \\
\text { mitochondria }\end{array}$ & ENO2P, pre-KARS2 & $\begin{array}{l}\text { Mitochondrial } \\
\text { import }\end{array}$ & Mitochondrial translation & $(62)$ \\
\hline & $5 S$ rRNA & $\begin{array}{l}\text { Cytoplasm, } \\
\text { mitochondria }\end{array}$ & $\begin{array}{l}\text { TFIIIA, RPL5, MRP } \\
\text { L18, Rhodanese }\end{array}$ & $\begin{array}{l}\text { Nuclear export/ } \\
\text { mitochondrial } \\
\text { import }\end{array}$ & & $(67,68,73)$ \\
\hline & MRP RNA (RMRP) & $\begin{array}{l}\text { Nucleus, } \\
\text { mitochondria }\end{array}$ & $\begin{array}{l}\text { HuR, CRM1, } \\
\text { GRSF1, PNPASE, } \\
\text { hTERT }\end{array}$ & $\begin{array}{l}\text { Nuclear export/ } \\
\text { mitochondrial } \\
\text { import }\end{array}$ & $\begin{array}{l}\text { Ribosomal RNA ( } 5.8 S) \\
\text { maturation, cell division, } \\
\text { mitochondrial RNA } \\
\text { processing, mitochondrial } \\
\text { DNA replication }\end{array}$ & $(17,19)$ \\
\hline & $\begin{array}{l}\text { RNase P RNA } \\
\text { (RPPH1) }\end{array}$ & $\begin{array}{l}\text { Nucleus, } \\
\text { mitochondria }\end{array}$ & PNPASE & $\begin{array}{l}\text { Mitochondrial } \\
\text { import }\end{array}$ & Unknown & $(17)$ \\
\hline & miRNAs (mitomiRs) & $\begin{array}{l}\text { Cytoplasm, } \\
\text { mitochondria }\end{array}$ & AGO2 & Unknown & $\begin{array}{l}\text { Mitochondrial gene } \\
\text { expression }\end{array}$ & $(14,15,105,112)$ \\
\hline
\end{tabular}

by binding the $5^{\prime}$ untranslated region (UTR) of B-cyclin (CLB2) mRNA and degrading CLB2 mRNA during mitosis (78). Human telomerase reverse transcriptase (hTERT) also associated with RMRP and synthesized a double-stranded RNA (dsRMRP) that was recognized by the endoribonuclease DICER1 and processed into short interfering (si) RNA (79).

There is strong evidence that $R M R P$ is also found in mammalian mitochondria $(17,19,23)$. The Clayton laboratory further proposed that $R M R P$ was involved in mitochondrial RNA processing as well as in mitochondrial DNA replication $(7,8)$, although these functions are not fully understood. PNPASE (polynucleotide phosphorylase or 3'-to-5' exoribonuclease and poly(A) polymerase) is a nuclear DNA-encoded protein that can be localized in the mitochondrial intermembrane space (IMS) (80-82). Recently, Wang et al. identified a novel function for mitochondrial PNPASE in regulating the import into the mitochondrial matrix of RNAs transcribed in the nucleus. Besides its impact on mitochondrial RNA processing, translation, and respiration, PNPASE can internalize RNAs including $R M R P$, $5 S$ rRNA, and RNase P RNA (RPPH1, described in the next section) from the IMS into the matrix. Interestingly, both $R M R P$ and $R P P H 1$ were shown to share a stem loop structure recognized by PNPASE, which is critical for their translocation (17) (Table 1, Fig. 1).

Very recently, Noh et al. proposed a molecular mechanism whereby RBPs contributed to the intracellular and the suborganelle-specific distribution of IncRNP complexes. Their findings revealed that $R M R P$ is exported from the nucleus to the cytosol by the RBP HuR through a CRM1 (chromosome region maintenance 1)-dependent nuclear export pathway. The exported $R M R P$ was targeted into the mitochondrial matrix through unknown mechanisms, but once in the matrix, a nuclear DNA-encoded, mitochondria-resident RBP, GRSF1 (G-rich RNA sequence-binding factor 1), interacted with the imported RMRP and the resulting IncRNP complex was proposed to contribute to mitochondrial function by affecting the OX-PHOS system and mitochondrial DNA replication (19) (Table 1, Fig. 1).

\section{RNase P RNA (H1 RNA, RPPH1)}

RNase P RNA from bacteria, archaea, and eukarya was initially characterized as a catalytic subunit of a ribozyme capable of removing the $5^{\prime}$ leader sequence from tRNA precursors in the absence of protein subunits (83-85). An endoribonuclease protein complex that cleaves the $E$. coli tRNA ${ }^{\mathrm{Tyr}}$ at the same site as bacterial RNase $\mathrm{P}$ was partially purified from mammalian mitochondria and was named mtRNase $P$, to distinguish it 
from its nuclear counterpart (nuRNase P) (86). Subsequent studies found that unlike mammalian nuRNase $\mathrm{P}$ and bacterial RNase $\mathrm{P}$, which include an RNA component, mammalian mtRNase $P$ does not $(13,87)$; however, other studies found an RNA similar to the $H 1$ RNA of nuRNase $\mathrm{P}$ bound to mammalian mtRNase $P(12,88)$. Despite controversy about its function, there is strong evidence that RNase $P$ RNA is imported into the mitochondrial matrix $(17,19,23)$ (Table 1 , Fig. 1).

\section{MITOCHONDRIA-DIRECTED MicroRNAs (mitomiRs)}

MicroRNAs (miRNAs) are small noncoding RNA molecules ( $\sim 22$ nucleotides long) that generally target and suppress mRNA stability and/or translation $(89,90)$. They are transcribed as primary (pri-)microRNAs, which are processed in the nucleus by the DROSHADGCR8 complex into precursor (pre-)microRNAs that are exported to the cytoplasm and further processed by DICER 1 into mature microRNAs. MicroRNAs associated with the RBP AGO2 (argonaute 2), a component of the RNA-induced silencing complex (RISC), are directed to target mRNAs with which they share partial sequence complementarity (91-94). MicroRNAs can potentially interact with a wide range of target mRNAs and thus have emerged as potent posttranscriptional regulators involved in several cellular processes, including survival, death, division, differentiation, and senescence (95-101). In turn, they impact upon physiologic processes and diseases, such as cancer, cardiovascular disease, neurodegeneration, aging, inflammation, and diabetes (101-103). The recent discovery of microRNAs in mitochondria has expanded the spectrum of possible posttranscriptional functions of microRNAs and has led to a search for mechanisms of microRNA translocation to mitochondria. In this section, we review some of these microRNAs and their impact on mitochondrial functions.

\section{MicroRNAs and AGO2 in mitochondria}

Generally, microRNAs function as the sequence specificityconferring component of the RISC, which suppresses the stability and/or translation of target mRNAs in the cytosol. The RISC includes multiple RBPs such as AGO, TRBP1, TRBP2, and GW182. Accumulating evidence indicates that microRNAs and AGO2 exist in mitochondria $(14,15,104)$, although the mechanisms through which microRNAs are imported into mitochondria as well as their functional impact are largely unknown. Pre-microRNAs have also been detected in mitochondria, suggesting that some aspects of microRNA biogenesis may occur in mitochondria (105-107). Moreover, the discovery of AGO2-bound microRNAs in mitochondria suggests that AGO2 may be important for the import of microRNAs into mitochondria possibly via co-import (108); interestingly, there is no evidence that other AGO proteins (e.g., AGO1 and AGO3) are imported to mitochondria (109). Crosslinking and immunoprecipitation (CLIP)-based analyses have further shown that AGO2 is associated with mitochondrial DNA-encoded transcripts (104). At present, the full mechanisms that govern these translocation events are not known.

\section{Function of mitomiRs}

The vast majority of microRNAs found in mitochondria, collectively known as mitomiRs (110), are encoded by nuclear DNA, although a few microRNAs (specifically, miR-1974, miR-1977, and miR-1978) are encoded by mitochondrial DNA (14). Although the functions of most mitomiRs are unknown, they have been suggested to play roles in cell survival, cell division, and energy metabolism, as well as in disease processes like cancer (111). Among the few mitomiRs that have been studied functionally, the nuclear DNA-encoded miR-181c was found to translocate into mitochondria and to regulate mitochondrial gene expression. In rodents, overexpression of miR-181c, which targeted and downregulated $m t-C O X 1$ (cytochrome c oxidase subunit 1) mRNA, led to higher ROS production, lower exercise capacity, and the appearance of cardiac dysfunction (112). Interestingly, the translocation of miR-1 into mitochondria with $\mathrm{AGO} 2$ led to increased translation of $\mathrm{COX} 1$ and the mitochondrial DNA-encoded NADH dehydrogenase 1 (ND1), suggesting that miR-1 coordinates gene expression networks in the cytoplasm and the mitochondria during muscle differentiation (109).

Next-generation sequencing (NGS) identified several microRNAs imported into mitochondria in $206 \rho^{\circ}$ cells, such as miR-181c-5p and miR-146a-5p, which had many potential targets within the mitochondria, including RNAs transcribed from nuclear DNA and from mitochondrial DNA $(112,113)$. The mitochondria-localized miR-34a was recently found to control the integrity of the blood-brain barrier in cultured cerebrovascular endothelial cells by lowering mitochondrial oxidative phosphorylation and the levels of adenosine triphosphate and cytochrome c (114). However, miR-34a was also detected in the cytosol and thus its effect on the integrity of the blood-brain barrier might include miR-34a actions on cytosolic mRNAs. In a mouse model of diabetic heart and in HL-1 cells, Jagannathan et al. identified a pool of mitomiRs including miR-378, which translocated into mitochondria following a diabetic insult and downregulated the mitochondrially encoded F0 component ATP6, essential for cardiac pump function (115).

Together, these studies provide evidence that a substantial portion of microRNAs are imported into mitochondria and may influence mitochondrial gene expression programs widely. MitomiRs can enhance and reduce the expression of mRNAs originated from mitochondrial and nuclear transcription and in turn affect mitochondrial metabolic activity and cell homeostasis. It will be important to elucidate the mechanisms that govern microRNA localization in mitochondria, including the transport and interaction factors (e.g., RBPs and long noncoding RNAs), the mitochondria localization signals that tag microRNAs to mitochondria (e.g., RNA motifs), and the mitochondrial 
transport machineries that mediate such transport. In addition, it will be critical to identify the mitochondrial interaction partners (AGO2 and likely other RBPs) that enable the mitochondrial functions of mitomiRs.

\section{CONCLUDING REMARKS}

We have summarized our knowledge of noncoding RNAs mobilized into and out of mitochondria as well as their function. We discuss specific areas that warrant immediate attention as the field progresses.

\section{RNP complexes implicated in mitochondrial RNA mobilization}

Recent advances in high-throughput sequencing technology have revealed vast numbers of IncRNAs expressed in cells (116) and follow-up studies have shown that they regulate gene expression programs transcriptionally, post-transcriptionally, and post-translationally (117-121). Indeed, most IncRNAs transcribed from nuclear DNA form IncRNA-protein complexes (IncRNPs) that are essential for their function in both the nucleus and the cytoplasm.

We reviewed mitochondrial IncRNAs derived from the mitochondrial genome (IncND5, IncND6, IncCyt b, SncmtRNA, ASncmtRNA1/2, and LIPCAR) and the nuclear genome (tRNAs, $5 S$ rRNA, MRP RNA, and RNase P RNA). For many IncRNAs examined in molecular detail, the interacting protein partners (RBPs) $(122,123)$ have been associated with their function. Future studies should examine whether specific RBPs associated with IncRNAs function as import/export factors. Besides specialized RBPs, there might be a basic machinery that controls RNA mobilization. This machinery employs ATP and appears to rely on factors located in the mitochondrial outer membrane (OM) as well as on core components of the protein import pathway, the TOM/TIM complex (60). An earlier study in yeast suggested that the yeast Tom20 and Tim44 complexes were involved in translocating the cytoplasmic tRNA ${ }^{\text {Lys }}$ into mitochondria (59). VDAC (voltage-dependent anion channel), an abundant structural protein in the OM, appeared to contribute to mitochondrial tRNA import in plants (124). It will be important to test in the near future if VDAC and/or other OM-associated proteins can recognize and capture cytosolic RNAs at the surface of mammalian mitochondria.

\section{Composition and function of the mitoribosome (mitochondrial ribosome)}

The mitochondrial ribosome (mitoribosome) has been identified in organisms from yeast to mammalian cells $(125,126)$, and was found to be poorly conserved in structure and composition among species. The mitoribosome is associated with the mitochondrial inner membrane, facing the matrix side, which enables it to insert highly hydrophobic nascent polypeptides easily upon translation $(22,127-130)$. Smirnov et al. proposed a model whereby the imported $5 S$ rRNA associated with the mitoribosomal large subunit (LSU), affecting mitochondrial translation efficiency (18) and helping to explain the high abundance of $5 S$ rRNAs found in mammalian mitochondria. However, a more recent study using cryo-EM provided alternative molecular evidence that mitoribosomal LSU has negligible or no $5 S$ rRNA (131). Considering its vital roles for maintaining mitochondrial biogenesis and functions, the structural and functional RNA component of the mitoribosome also deserves in-depth investigation.

\section{Impact of mitochondrial RNA mobilization on cellular homeostasis and disease}

Optimal mitochondrial activity is necessary for cell homeostasis. Accordingly, mitochondrial dysfunction has been linked to chronic neurodegenerative disorders including Alzheimer's disease (AD), associated with the deposition of toxic $A \beta$ peptide and DNA damage caused by defective base excision repair (132-134), and Parkinson's disease, caused by genetic alterations of PINK1, Parkin, DJ-1, and $\alpha$-Synuclein (135-138). In addition, increasing evidence suggests that mitochondrial dysfunction contributes to cardiovascular disease (139, 140), muscle atrophy (141), insulin resistance (142), chronic obstructive pulmonary disease (143), cancer cachexia (144), and neuromuscular disorders (145). Mitochondrial dysfunction associated with damage and mutations of the mitochondrial DNA has been linked to accelerated aging and age-related disease $(146,147)$.

As previously hypothesized, mitochondria-directed RNA can be used as an endogenous vector that is effectively mobilized into mitochondria, carrying inserted heterologous sequences. Theoretically, it seems possible to introduce a particular RNA sequence which is complementary to the mutated region of mitochondrial DNA, thereby lowering the replication efficiency and clearing the 'bad' mitochondria from cells (148). In fact, interventions devised to eliminate pathogenic mitochondrial DNA, involving the creation of a chimeric RNA bearing a mitochondrial import signal and a sequence proximal to the mitochondrial DNA mutation site, were recently reported (149). These and other strategies are under consideration as we develop molecular and pharmacological methods to intervene in disease processes linked to aberrant mitochondrial function.

\section{ACKNOWLEDGEMENTS}

This work was supported in full by the National Institute on Aging Intramural Research Program of the National Institutes of Health.

\section{CONFLICTS OF INTEREST}

The authors have no conflicting financial interests. 


\section{REFERENCES}

1. Gray MW, Burger G and Lang BF (1999) Mitochondrial evolution. Science 283, 1476-1481

2. Lane N and Martin W (2010) The energetics of genome complexity. Nature 467, 929-934

3. Neupert W and Herrmann JM (2007) Translocation of proteins into mitochondria. Annu Rev Biochem 76, 723-749

4. Chacinska A, Koehler CM, Milenkovic D, Lithgow T and Pfanner N (2009) Importing mitochondrial proteins: machineries and mechanisms. Cell 138, 628-644

5. Schmidt O, Pfanner N and Meisinger C (2010) Mitochondrial protein import: from proteomics to functional mechanisms. Nat Rev Mol Cell Biol 11, 655-667

6. Suyama Y (1967) The origins of mitochondrial ribonucleic acids in Tetrahymena pyriformis. Biochemistry 6, 28292839

7. Chang DD and Clayton DA (1989) Mouse RNAase MRP RNA is encoded by a nuclear gene and contains a decamer sequence complementary to a conserved region of mitochondrial RNA substrate. Cell 56, 131-139

8. Chang DD and Clayton DA (1987) A mammalian mitochondrial RNA processing activity contains nucleusencoded RNA. Science 235, 1178-1184

9. Yoshionari S, Koike T, Yokogawa T et al (1994) Existence of nuclear-encoded 5S-rRNA in bovine mitochondria. FEBS Lett 338, 137-142

10. Kiss T and Filipowicz W (1992) Evidence against a mitochondrial location of the 7-2/MRP RNA in mammalian cells. Cell 70, 11-16

11. Magalhaes PJ, Andreu AL and Schon EA (1998) Evidence for the presence of 5S rRNA in mammalian mitochondria. Mol Biol Cell 9, 2375-2382

12. Puranam RS and Attardi G (2001) The RNase P associated with HeLa cell mitochondria contains an essential RNA component identical in sequence to that of the nuclear RNase P. Mol Cell Biol 21, 548-561

13. Holzmann I, Frank $P$, Loffler $E$, Bennett $K L$, Gerner $C$ and Rossmanith W (2008) RNase P without RNA: identification and functional reconstitution of the human mitochondrial tRNA processing enzyme. Cell 135, 462-474

14. Bandiera S, Ruberg S, Girard M et al (2011) Nuclear outsourcing of RNA interference components to human mitochondria. PLoS One 6, e20746

15. Sripada $L$, Tomar D, Prajapati $P$, Singh $R$, Singh $A K$ and Singh R (2012) Systematic analysis of small RNAs associated with human mitochondria by deep sequencing: detailed analysis of mitochondrial associated miRNA. PLoS One 7, e44873

16. Kamenski P, Kolesnikova O, Jubenot $\mathrm{V}$ et al (2007) Evidence for an adaptation mechanism of mitochondrial translation via tRNA import from the cytosol. Mol Cell $26,625-637$

17. Wang G, Chen HW, Oktay $Y$ et al (2010) PNPASE regulates RNA import into mitochondria. Cell 142, 456-467

18. Smirnov A, Entelis N, Martin RP and Tarassov I (2011) Biological significance of $5 \mathrm{~S}$ rRNA import into human mitochondria: role of ribosomal protein MRP-L18. Genes Dev 25, 1289-1305

19. Noh JH, Kim KM, Abdelmohsen $\mathrm{K}$ et al (2016) HuR and GRSF1 modulate the nuclear export and mitochondrial localization of the IncRNA RMRP. Genes Dev 30, 1224-1239

20. O'Brien TW (2002) Evolution of a protein-rich mitochondrial ribosome: implications for human genetic disease. Gene 286, 73-79

21. Ojala D, Montoya J and Attardi G (1981) tRNA punctuation model of RNA processing in human mitochondria. Nature 290, 470-474

22. Attardi G and Ojala D (1971) Mitochondrial ribssome in HeLa cells. Nat New Biol 229, 133-136

23. Mercer TR, Neph S, Dinger ME et al (2011) The human mitochondrial transcriptome. Cell 146, 645-658

24. Rackham O, Shearwood AM, Mercer TR, Davies SM, Mattick JS and Filipovska A (2011) Long noncoding RNAs are generated from the mitochondrial genome and regulated by nuclear-encoded proteins. RNA 17, 20852093

25. Sanchez MI, Mercer TR, Davies SM et al (2011) RNA processing in human mitochondria. Cell Cycle 10, 2904-2916

26. Villegas J, Burzio V, Villota C et al (2007) Expression of a novel non-coding mitochondrial RNA in human proliferating cells. Nucleic Acids Res 35, 7336-7347

27. Villegas J, Zarraga AM, Muller I et al (2000) A novel chimeric mitochondrial RNA localized in the nucleus of mouse sperm. DNA Cell Biol 19, 579-588

28. Burzio VA, Villota C, Villegas J et al (2009) Expression of a family of noncoding mitochondrial RNAs distinguishes normal from cancer cells. Proc Natl Acad Sci U S A 106, 9430-9434

29. Landerer E, Villegas J, Burzio VA et al (2011) Nuclear localization of the mitochondrial ncRNAs in normal and cancer cells. Cell Oncol (Dordr) 34, 297-305

30. Bianchessi V, Badi I, Bertolotti M et al (2015) The mitochondrial IncRNA ASncmtRNA-2 is induced in aging and replicative senescence in Endothelial Cells. J Mol Cell Cardiol 81, 62-70

31. Kumarswamy R, Bauters C, Volkmann I et al (2014) Circulating long noncoding RNA, LIPCAR, predicts survival in patients with heart failure. Circ Res 114, 1569-1575

32. Entelis NS, Kolesnikova OA, Dogan S, Martin RP and Tarassov IA (2001) 5 S rRNA and tRNA import into human mitochondria. Comparison of in vitro requirements. J Biol Chem 276, 45642-45653

33. Li K, Smagula CS, Parsons WJ et al (1994) Subcellular partitioning of MRP RNA assessed by ultrastructural and biochemical analysis. J Cell Biol 124, 871-882

34. Martin RP, Schneller JM, Stahl AJ and Dirheimer G (1977) Study of yeast mitochondrial tRNAs by twodimensional polyacrylamide gel electrophoresis: characterization of isoaccepting species and search for imported cytoplasmic tRNAs. Nucleic Acids Res 4, 3497-3510

35. Simpson AM, Suyama Y, Dewes H, Campbell DA and Simpson L (1989) Kinetoplastid mitochondria contain functional tRNAs which are encoded in nuclear DNA 
and also contain small minicircle and maxicircle transcripts of unknown function. Nucleic Acids Res 17, 5427-5445

36. Hancock K and Hajduk SL (1990) The mitochondrial tRNAs of Trypanosoma brucei are nuclear encoded. J Biol Chem 265, 19208-19215

37. Mottram JC, Bell SD, Nelson RG and Barry JD (1991) tRNAs of Trypanosoma brucei. Unusual gene organization and mitochondrial importation. J Biol Chem 266, 18313-18317

38. Lye LF, Chen DH and Suyama Y (1993) Selective import of nuclear-encoded tRNAs into mitochondria of the protozoan Leishmania tarentolae. Mol Biochem Parasitol 58, 233-245

39. Schneider A, Martin J and Agabian N (1994) A nuclear encoded tRNA of Trypanosoma brucei is imported into mitochondria. Mol Cell Biol 14, 2317-2322

40. Rusconi CP and Cech TR (1996) Mitochondrial import of only one of three nuclear-encoded glutamine tRNAs in Tetrahymena thermophila. EMBO J 15, 3286-3295

41. Martin RP, Schneller JM, Stahl AJ and Dirheimer G (1979) Import of nuclear deoxyribonucleic acid coded lysine-accepting transfer ribonucleic acid (anticodon C-U-U) into yeast mitochondria. Biochemistry 18, 46004605

42. Tarassov IA and Martin RP (1996) Mechanisms of tRNA import into yeast mitochondria: an overview. Biochimie $78,502-510$

43. Marechal-Drouard L, Weil JH and Guillemaut P (1988) Import of several tRNAs from the cytoplasm into the mitochondria in bean Phaseolus vulgaris. Nucleic Acids Res 16, 4777-4788

44. Dietrich A, Small I, Cosset A, Weil JH and MarechalDrouard L (1996) Editing and import: strategies for providing plant mitochondria with a complete set of functional transfer RNAs. Biochimie 78, 518-529

45. Akashi K, Sakurai K, Hirayama J, Fukuzawa H and Ohyama K (1996) Occurrence of nuclear-encoded tRNAlle in mitochondria of the liverwort Marchantia polymorpha. Curr Genet 30, 181-185

46. Chen HC, Viry-Moussaid M, Dietrich A and Wintz $H$ (1997) Evolution of a mitochondrial tRNA PHE gene in A. thaliana: import of cytosolic tRNA PHE into mitochondria. Biochem Biophys Res Commun 237, 432-437

47. Glover KE, Spencer DF and Gray MW (2001) Identification and structural characterization of nucleus-encoded transfer RNAs imported into wheat mitochondria. J Biol Chem 276, 639-648

48. Brubacher-Kauffmann S, Marechal-Drouard L, Cosset A, Dietrich A and Duchene AM (1999) Differential import of nuclear-encoded tRNAGly isoacceptors into solanum Tuberosum mitochondria. Nucleic Acids Res 27, 20372042

49. Dorner $M$, Altmann $M$, Paabo S and Morl M (2001) Evidence for import of a lysyl-tRNA into marsupial mitochondria. Mol Biol Cell 12, 2688-2698

50. Schneider A (2011) Mitochondrial tRNA import and its consequences for mitochondrial translation. Annu Rev Biochem 80, 1033-1053

51. Tarassov I, Kamenski P, Kolesnikova O et al (2007)
Import of nuclear DNA-encoded RNAs into mitochondria and mitochondrial translation. Cell Cycle 6, 2473-2477

52. Kolesnikova OA, Entelis NS, Jacquin-Becker $\mathrm{C}$ et al (2004) Nuclear DNA-encoded tRNAs targeted into mitochondria can rescue a mitochondrial DNA mutation associated with the MERRF syndrome in cultured human cells. Hum Mol Genet 13, 2519-2534

53. Kolesnikova OA, Entelis NS, Mireau H, Fox TD, Martin RP and Tarassov IA (2000) Suppression of mutations in mitochondrial DNA by tRNAs imported from the cytoplasm. Science 289, 1931-1933

54. Tarassov IA and Entelis NS (1992) Mitochondriallyimported cytoplasmic tRNA(Lys)(CUU) of Saccharomyces cerevisiae: in vivo and in vitro targetting systems. Nucleic Acids Res 20, 1277-1281

55. Rubio MA, Rinehart JJ, Krett B et al (2008) Mammalian mitochondria have the innate ability to import tRNAs by a mechanism distinct from protein import. Proc Natl Acad Sci U S A 105, 9186-9191

56. Brandina I, Graham J, Lemaitre-Guillier C et al (2006) Enolase takes part in a macromolecular complex associated to mitochondria in yeast. Biochim Biophys Acta 1757, 1217-1228

57. Entelis N, Brandina I, Kamenski $P$, Krasheninnikov IA, Martin RP and Tarassov I (2006) A glycolytic enzyme, enolase, is recruited as a cofactor of tRNA targeting toward mitochondria in Saccharomyces cerevisiae. Genes Dev 20, 1609-1620

58. Kamenski P, Smirnova E, Kolesnikova O et al (2010) tRNA mitochondrial import in yeast: Mapping of the import determinants in the carrier protein, the precursor of mitochondrial lysyl-tRNA synthetase. Mitochondrion 10, 284-293

59. Tarassov I, Entelis N and Martin RP (1995) Mitochondrial import of a cytoplasmic lysine-tRNA in yeast is mediated by cooperation of cytoplasmic and mitochondrial lysyltRNA synthetases. EMBO J 14, 3461-3471

60. Tarassov I, Entelis N and Martin RP (1995) An intact protein translocating machinery is required for mitochondrial import of a yeast cytoplasmic tRNA. J Mol Biol 245, 315-323

61. Kolesnikova O, Kazakova H, Comte C et al (2010) Selection of RNA aptamers imported into yeast and human mitochondria. RNA 16, 926-941

62. Gowher A, Smirnov A, Tarassov I and Entelis N (2013) Induced tRNA import into human mitochondria: implication of a host aminoacyl-tRNA-synthetase. PLoS One 8, e66228

63. Eilers M and Schatz G (1986) Binding of a specific ligand inhibits import of a purified precursor protein into mitochondria. Nature 322, 228-232

64. Entelis NS, Kieffer S, Kolesnikova OA, Martin RP and Tarassov IA (1998) Structural requirements of tRNALys for its import into yeast mitochondria. Proc Natl Acad Sci U S A 95, 2838-2843

65. Vestweber D and Schatz G (1989) DNA-protein conjugates can enter mitochondria via the protein import pathway. Nature 338, 170-172

66. Nierlich DP (1982) Fragmentary 5S rRNA gene in the human mitochondrial genome. Mol Cell Biol 2, 207-209 
67. Pelham HR and Brown DD (1980) A specific transcription factor that can bind either the 5S RNA gene or 5S RNA. Proc Natl Acad Sci U S A 77, 4170-4174

68. Guddat U, Bakken $A H$ and Pieler $T$ (1990) Proteinmediated nuclear export of RNA: 5S rRNA containing small RNPs in xenopus oocytes. Cell 60, 619-628

69. Chan YL, Lin A, McNally J and Wool IG (1987) The primary structure of rat ribosomal protein L5. A comparison of the sequence of amino acids in the proteins that interact with $5 \mathrm{~S}$ rRNA. J Biol Chem 262, 1287912886

70. Steitz JA, Berg C, Hendrick JP et al (1988) A 5S rRNA/L5 complex is a precursor to ribosome assembly in mammalian cells. J Cell Biol 106, 545-556

71. Rudt F and Pieler T (1996) Cytoplasmic retention and nuclear import of 5S ribosomal RNA containing RNPs. EMBO J 15, 1383-1391

72. Smirnov A, Tarassov I, Mager-Heckel AM et al (2008) Two distinct structural elements of 5S rRNA are needed for its import into human mitochondria. RNA 14, 749-759

73. Smirnov A, Comte C, Mager-Heckel AM et al (2010) Mitochondrial enzyme rhodanese is essential for $5 \mathrm{~S}$ ribosomal RNA import into human mitochondria. J Biol Chem 285, 30792-30803

74. Ridanpaa M, van Eenennaam H, Pelin K et al (2001) Mutations in the RNA component of RNase MRP cause a pleiotropic human disease, cartilage-hair hypoplasia. Cell 104, 195-203

75. Hermanns P, Bertuch AA, Bertin TK et al (2005) Consequences of mutations in the non-coding RMRP RNA in cartilage-hair hypoplasia. Hum Mol Genet 14, 3723-3740

76. Chu S, Archer RH, Zengel JM and Lindahl L (1994) The RNA of RNase MRP is required for normal processing of ribosomal RNA. Proc Natl Acad Sci U S A 91, 659-663

77. Schmitt ME and Clayton DA (1993) Nuclear RNase MRP is required for correct processing of pre-5.8S rRNA in Saccharomyces cerevisiae. Mol Cell Biol 13, 7935-7941

78. Gill T, Cai T, Aulds J, Wierzbicki S and Schmitt ME (2004) RNase MRP cleaves the CLB2 mRNA to promote cell cycle progression: novel method of mRNA degradation. Mol Cell Biol 24, 945-953

79. Maida Y, Yasukawa M, Furuuchi M et al (2009) An RNA-dependent RNA polymerase formed by TERT and the RMRP RNA. Nature 461, 230-235

80. Chen HW, Rainey RN, Balatoni CE et al (2006) Mammalian polynucleotide phosphorylase is an intermembrane space RNase that maintains mitochondrial homeostasis. Mol Cell Biol 26, 8475-8487

81. Chen HW, Koehler CM and Teitell MA (2007) Human polynucleotide phosphorylase: location matters. Trends Cell Biol 17, 600-608

82. Rainey RN, Glavin JD, Chen HW, French SW, Teitell MA and Koehler CM (2006) A new function in translocation for the mitochondrial i-AAA protease Yme1: import of polynucleotide phosphorylase into the intermembrane space. Mol Cell Biol 26, 8488-8497

83. Guerrier-Takada C, Gardiner K, Marsh T, Pace N and Altman S (1983) The RNA moiety of ribonuclease $P$ is the catalytic subunit of the enzyme. Cell 35, 849-857

84. Pannucci JA, Haas ES, Hall TA, Harris JK and Brown JW (1999) RNase P RNAs from some Archaea are catalytically active. Proc Natl Acad Sci U S A 96, 7803-7808

85. Kikovska E, Svard SG and Kirsebom LA (2007) Eukaryotic RNase P RNA mediates cleavage in the absence of protein. Proc Natl Acad Sci U S A 104, 2062-2067

86. Doersen CJ, Guerrier-Takada C, Altman S and Attardi G (1985) Characterization of an RNase P activity from HeLa cell mitochondria. Comparison with the cytosol RNase P activity. J Biol Chem 260, 5942-5949

87. Rossmanith W and Karwan RM (1998) Characterization of human mitochondrial RNase P: novel aspects in tRNA processing. Biochem Biophys Res Commun 247, 234241

88. Bartkiewicz M, Gold $\mathrm{H}$ and Altman S (1989) Identification and characterization of an RNA molecule that copurifies with RNase P activity from HeLa cells. Genes Dev 3, 488-499

89. Huntzinger E and Izaurralde E (2011) Gene silencing by microRNAs: contributions of translational repression and mRNA decay. Nat Rev Genet 12, 99-110

90. Filipowicz W, Bhattacharyya SN and Sonenberg N (2008) Mechanisms of post-transcriptional regulation by microRNAs: are the answers in sight? Nat Rev Genet 9, 102-114

91. Fabian MR, Sonenberg N and Filipowicz W (2010) Regulation of mRNA translation and stability by microRNAs. Annu Rev Biochem 79, 351-379

92. Bartel DP (2009) MicroRNAs: target recognition and regulatory functions. Cell 136, 215-233

93. Kim VN, Han J and Siomi MC (2009) Biogenesis of small RNAs in animals. Nat Rev Mol Cell Biol 10, 126-139

94. Newman MA and Hammond SM (2010) Emerging paradigms of regulated microRNA processing. Genes Dev 24, 1086-1092

95. Kloosterman WP and Plasterk RH (2006) The diverse functions of microRNAs in animal development and disease. Dev Cell 11, 441-450

96. Lingor P (2010) Regulation of Cell Death and Survival by RNA Interference - The Roles of miRNA and siRNA; in Apoptosome: An up-and-coming therapeutical tool, Cecconi F and D'Amelio M (eds.), 95-117, Springer Netherlands, Dordrecht

97. Catalanotto C, Cogoni C and Zardo G (2016) MicroRNA in Control of Gene Expression: An Overview of Nuclear Functions. Int J Mol Sci 17

98. Yao S (2016) MicroRNA biogenesis and their functions in regulating stem cell potency and differentiation. Biol Proced Online 18, 8

99. Bushati $N$ and Cohen SM (2007) microRNA functions. Annu Rev Cell Dev Biol 23, 175-205

100. Abdelmohsen K and Gorospe M (2015) Noncoding RNA control of cellular senescence. Wiley Interdiscip Rev RNA 6, 615-629

101. Rottiers $V$ and Naar AM (2012) MicroRNAs in metabolism and metabolic disorders. Nat Rev Mol Cell Biol 13, 239-250

102. Shah MY, Ferrajoli A, Sood AK, Lopez-Berestein G and 
Calin GA (2016) microRNA Therapeutics in Cancer - An Emerging Concept. EBioMedicine 12, 34-42

103. Saeidimehr S, Ebrahimi A, Saki N, Goodarzi P and Rahim F (2016) MicroRNA-Based Linkage between Aging and Cancer: from Epigenetics View Point. Cell J $18,117-126$

104. Das S, Ferlito M, Kent OA et al (2012) Nuclear miRNA regulates the mitochondrial genome in the heart. Circ Res 110, 1596-1603

105. Sripada L, Tomar D and Singh R (2012) Mitochondria: one of the destinations of miRNAs. Mitochondrion 12, 593-599

106. Bienertova-Vasku J, Sana J and Slaby O (2013) The role of microRNAs in mitochondria in cancer. Cancer Lett 336, 1-7

107. Barrey E, Saint-Auret G, Bonnamy B, Damas D, Boyer O and Gidrol X (2011) Pre-microRNA and mature microRNA in human mitochondria. PLoS One 6, e20220

108. Srinivasan $\mathrm{H}$ and Das S (2015) Mitochondrial miRNA (MitomiR): a new player in cardiovascular health. Can J Physiol Pharmacol 93, 855-861

109. Zhang X, Zuo X, Yang B et al (2014) MicroRNA directly enhances mitochondrial translation during muscle differentiation. Cell 158, 607-619

110. Bandiera S, Mategot R, Girard M, Demongeot J and Henrion-Caude A (2013) MitomiRs delineating the intracellular localization of microRNAs at mitochondria. Free Radic Biol Med 64, 12-19

111. Duarte FV, Palmeira CM and Rolo AP (2014) The Role of microRNAs in Mitochondria: Small Players Acting Wide. Genes (Basel) 5, 865-886

112. Das S, Bedja D, Campbell N et al (2014) miR-181c regulates the mitochondrial genome, bioenergetics, and propensity for heart failure in vivo. PLoS One 9, e96820

113. Dasgupta N, Peng Y, Tan Z, Ciraolo G, Wang D and Li R (2015) miRNAs in mtDNA-less cell mitochondria. Cell Death Discov 1, 15004

114. Bukeirat M, Sarkar SN, Hu H, Quintana DD, Simpkins JW and Ren X (2016) MiR-34a regulates blood-brain barrier permeability and mitochondrial function by targeting cytochrome c. J Cereb Blood Flow Metab 36, 387-392

115. Jagannathan R, Thapa D, Nichols CE et al (2015) Translational Regulation of the Mitochondrial Genome Following Redistribution of Mitochondrial MicroRNA in the Diabetic Heart. Circ Cardiovasc Genet 8, 785-802

116. Guttman M and Rinn JL (2012) Modular regulatory principles of large non-coding RNAs. Nature 482, 339346

117. Kretz M, Siprashvili Z, Chu C et al (2013) Control of somatic tissue differentiation by the long non-coding RNA TINCR. Nature 493, 231-235

118. Guttman M, Amit I, Garber M et al (2009) Chromatin signature reveals over a thousand highly conserved large non-coding RNAs in mammals. Nature 458, 223-227

119. Khalil AM, Guttman M, Huarte M et al (2009) Many human large intergenic noncoding RNAs associate with chromatin-modifying complexes and affect gene expression. Proc Natl Acad Sci U S A 106, 11667-11672

120. Rinn JL, Kertesz M, Wang JK et al (2007) Functional demarcation of active and silent chromatin domains in human HOX loci by noncoding RNAs. Cell 129, 1311-1323

121. Mattick JS and Makunin IV (2006) Non-coding RNA. Hum Mol Genet 15 Spec No 1, R17-29

122. Carpenter S, Aiello D, Atianand MK et al (2013) A long noncoding RNA mediates both activation and repression of immune response genes. Science 341, 789-792

123. Huarte M, Guttman M, Feldser D et al (2010) A large intergenic noncoding RNA induced by p53 mediates global gene repression in the p53 response. Cell 142, 409-419

124. Salinas T, Duchene AM, Delage L et al (2006) The voltage-dependent anion channel, a major component of the tRNA import machinery in plant mitochondria. Proc Natl Acad Sci U S A 103, 18362-18367

125. O'Brien TW (1971) The general occurrence of $55 \mathrm{~S}$ ribosomes in mammalian liver mitochondria. J Biol Chem 246, 3409-3417

126. Grivell LA, Reijnders L and Borst P (1971) Isolation of yeast mitochondrial ribosomes highly active in protein synthesis. Biochim Biophys Acta 247, 91-103

127. Sharma MR, Booth TM, Simpson L, Maslov DA and Agrawal RK (2009) Structure of a mitochondrial ribosome with minimal RNA. Proc Natl Acad Sci U S A 106, 9637-9642

128. Sharma MR, Koc EC, Datta PP, Booth TM, Spremulli LL and Agrawal RK (2003) Structure of the mammalian mitochondrial ribosome reveals an expanded functional role for its component proteins. Cell 115, 97-108

129. Smits P, Smeitink JA, van den Heuvel LP, Huynen MA and Ettema TJ (2007) Reconstructing the evolution of the mitochondrial ribosomal proteome. Nucleic Acids Res 35, 4686-4703

130. Mears JA, Sharma MR, Gutell RR et al (2006) A structural model for the large subunit of the mammalian mitochondrial ribosome. J Mol Biol 358, 193-212

131. Agrawal RK and Sharma MR (2012) Structural aspects of mitochondrial translational apparatus. Curr Opin Struct Biol 22, 797-803

132. Cha MY, Han SH, Son SM et al (2012) Mitochondriaspecific accumulation of amyloid beta induces mitochondrial dysfunction leading to apoptotic cell death. PLoS One 7, e34929

133. Reddy PH (2009) Amyloid beta, mitochondrial structural and functional dynamics in Alzheimer's disease. Exp Neurol 218, 286-292

134. Canugovi C, Shamanna RA, Croteau DL and Bohr VA (2014) Base excision DNA repair levels in mitochondrial lysates of Alzheimer's disease. Neurobiol Aging 35, 1293-1300

135. Marongiu R, Spencer B, Crews L et al (2009) Mutant Pink1 induces mitochondrial dysfunction in a neuronal cell model of Parkinson's disease by disturbing calcium flux. J Neurochem 108, 1561-1574

136. Mortiboys $\mathrm{H}$, Thomas KJ, Koopman WJ et al (2008) Mitochondrial function and morphology are impaired in parkin-mutant fibroblasts. Ann Neurol 64, 555-565

137. Canet-Aviles RM, Wilson MA, Miller DW et al (2004) The Parkinson's disease protein DJ-1 is neuroprotective 
due to cysteine-sulfinic acid-driven mitochondrial localization. Proc Natl Acad Sci U S A 101, 9103-9108

138. Devi L, Raghavendran V, Prabhu BM, Avadhani NG and Anandatheerthavarada HK (2008) Mitochondrial import and accumulation of alpha-synuclein impair complex I in human dopaminergic neuronal cultures and Parkinson disease brain. J Biol Chem 283, 9089-9100

139. Ide T, Tsutsui $H$, Hayashidani $S$ et al (2001) Mitochondrial DNA damage and dysfunction associated with oxidative stress in failing hearts after myocardial infarction. Circ Res 88, 529-535

140. Ballinger SW, Patterson C, Knight-Lozano CA et al (2002) Mitochondrial integrity and function in atherogenesis. Circulation 106, 544-549

141. Romanello V, Guadagnin E, Gomes L et al (2010) Mitochondrial fission and remodelling contributes to muscle atrophy. EMBO J 29, 1774-1785

142. Crescenzo R, Bianco F, Mazzoli A, Giacco A, Liverini G and lossa $S$ (2014) Mitochondrial efficiency and insulin resistance. Front Physiol 5, 512

143. Mathur S, Brooks D and Carvalho CR (2014) Structural alterations of skeletal muscle in copd. Front Physiol 5, 104
144. Antunes F, Corazzari M, Pereira G, Fimia GM, Piacentini $M$ and Smaili S (2016) Fasting boosts sensitivity of human skin melanoma to cisplatin-induced cell death. Biochem Biophys Res Commun [Epub ahead of print]

145. Katsetos CD, Anni $\mathrm{H}$ and Draber P (2013) Mitochondrial dysfunction in gliomas. Semin Pediatr Neurol 20, 216-227

146. Trifunovic A, Wredenberg A, Falkenberg M et al (2004) Premature ageing in mice expressing defective mitochondrial DNA polymerase. Nature 429, 417-423

147. Sastre J, Pallardo FV, Garcia de la Asuncion J and Vina J (2000) Mitochondria, oxidative stress and aging. Free Radic Res 32, 189-198

148. Smirnov AV, Entelis NS, Krasheninnikov IA, Martin R and Tarassov IA (2008) Specific features of 5S rRNA structure - its interactions with macromolecules and possible functions. Biochemistry (Mosc) 73, 1418-1437

149. Tonin Y, Heckel AM, Vysokikh M et al (2014) Modeling of antigenomic therapy of mitochondrial diseases by mitochondrially addressed RNA targeting a pathogenic point mutation in mitochondrial DNA. J Biol Chem 289, 13323-13334 